Access to this work was provided by the University of Maryland, Baltimore County (UMBC)

ScholarWorks@UMBC digital repository on the Maryland Shared Open Access (MD-SOAR) platform.

Please provide feedback

Please support the ScholarWorks@UMBC repository by emailing scholarworks-group@umbc.edu and telling us what having access to this work means to you and why it's important to you. Thank you. 


\title{
Cnoidal Waves in Microresonators
}

\author{
Zhen Qi, Giuseppe D'Aguanno, and Curtis R. Menyuk \\ University of Maryland, Baltimore County, 1000 Hilltop Circle, Baltimore, MD 21250, USA \\ zhenqi1@umbc.edu
}

\begin{abstract}
Cnoidal waves are the periodic analogs of solitons. Like solitons, they can be generated in microresonators and correspond to frequency combs. We describe their properties and potential uses.
\end{abstract}

OCIS codes: (230.5750) Resonators; (190.5530) Pulse propagation and temporal solitons; (190.4380) Nonlinear optics, four-wave mixing.

High-Q microresonators with a Kerr nonlinearity that is pumped by continuous-wave laser light can produce frequency combs. Broadband combs that can achieve nearly an octave of bandwidth have important potential applications to metrology and high-resolution spectroscopy and have recently attracted a large amount of attention [1,2]. However, narrowband combs also have important potential applications, including the nonlinear generation of new frequencies [3], and - because the comb lines of narrowband combs can be entangled — quantum networking [4]. Soliton generation appears at present to be the best path toward achieving an octave-spanning comb [1,2]. Solitons are not always easy to obtain, and it has been found computationally that periodic solutions will often appear instead in a microresonator $[5,6]$. Thus, it is important to understand the circumstances under which these periodic solutions appear and whether they can be used to create a broadband comb.

Here, we show that cnoidal wave solutions to the Lugiato-Lefever equation exist with a non-zero pump and zerodamping that can be expressed in terms of Jacobi elliptic functions [7] and that are analogous to the soliton solutions that Matsko et al. [8] found. Like the soliton solutions, these solutions are unstable, but become stable when the damping rate becomes sufficiently large. Like solitons, cnoidal wave solutions — also referred to as Turing rolls when they are stable - develop a chirp when the damping is non-zero and do not have an exactly analytical expression. However, the exact cnoidal wave solutions when the damping is zero are characterized by an exponential falloff in the envelope of the comb spectrum, and we find that this exponential falloff is still present when the damping is non-zero. Due to this exponential falloff, these cnoidal wave solutions are not suitable for broadband comb generation, but are well-suited to narrowband comb applications, where limiting the number of comb lines and precisely controlling their amplitudes is desirable.

The starting point of our analysis is the Lugiato-Lefever equation, which in its normalized form may be written

$$
i \frac{\partial \psi}{\partial t}+\frac{\partial^{2} \psi}{\partial x^{2}}-\psi+2|\psi|^{2} \psi=-i \alpha \psi-h
$$

where $\psi$ is the field envelope, $t$ is the normalized time, $x$ is the retarded and normalized azimuthal coordinate, $\alpha$ is the normalized damping rate, and $h$ is the normalized pump strength. When $\alpha=0$, this equation has solutions in terms of the Jacobi elliptic function $\operatorname{dn}\left[(x / g) \mid k^{2}\right]$ that may be written

$$
\psi(x)=\psi_{0}\left(1+\frac{A_{1} \mathrm{dn}\left[(x / g) \mid k^{2}\right]}{\operatorname{dn}\left[(x / g) \mid k^{2}\right]+A_{2}}\right),
$$

where $\psi_{0}, g, A_{1}$, and $A_{2}$ are all expressible as functions of $h$ and the Jacobi modulus $k^{2}$. A similar solution also exists in which $\mathrm{cn}\left[(x / g) \mid k^{2}\right]$ replaces $\operatorname{dn}\left[(x / g) \mid k^{2}\right]$. When the modulus $k \rightarrow 1$, both these solutions reduce to the soliton solution that Matsko et al. [8] found. When $\alpha \neq 0$, we can no longer find the stationary solutions analytically. Instead, we can find them when they are stable by numerically integrating Eq. (1) using a split-step algorithm with the initial conditions being the solution of Eq. (2). In Fig. 1 we show an example of these solutions.

Figure 1(a) shows $|\psi(x)|^{2}$ (dashed line) for a solution of Eq. (2) with $k=0.76$ and $h=0.8$, these parameters correspond to a periodicity of 5. This solution is compared to the numerical solution of Eq. (1) with $\alpha=0.5$ and same pump strength. Fig. 1(b) shows their Fourier spectra. Figures 1(c) and 1(d) show another case with the same parameters except that $\alpha=0.7$. In both cases, we find an exponential decay of the envelope of the Fourier spectrum. We note that the exponential decay of the envelope increases when $\alpha$ increases. To demonstrate that these cnoidal wave solutions are accessible from a broader set of initial conditions than the analytic cnoidal wave solutions at 

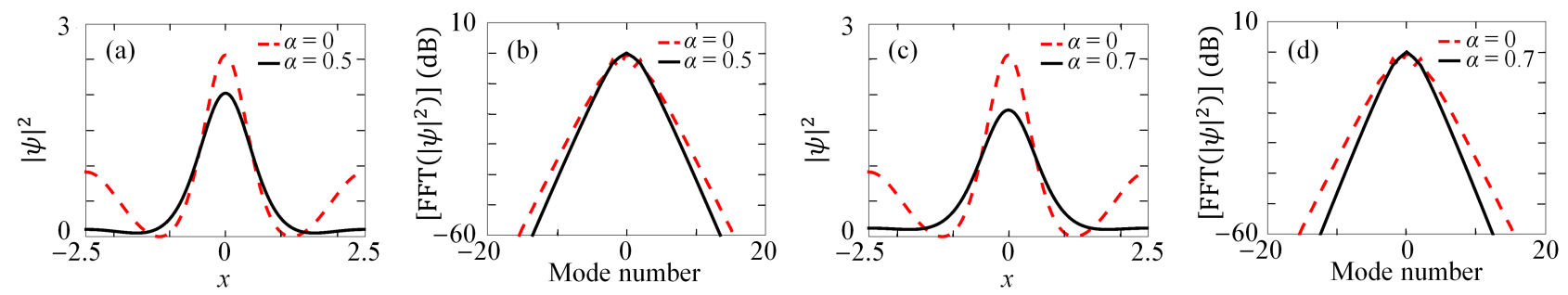

Fig. 1. (a) $|\psi(x)|^{2}$ (dashed line) for a solution of Eq. (2) with $k=0.76$ and $h=0.8$ corresponding to a period of 5. This solution is compared with the numerical solution of Eq. (1) with $\alpha=0.5$ and same pump strength (continuous line). (b) Fourier spectra of the modes. (c) Same as in (a) for the same parameters except that $\alpha=0.7$. (d) Fourier spectra of the modes.

$\alpha=0$, we have solved Eq. (1) numerically using two impulsive functions as the initial conditions. The first impulsive function is $10^{5}$ at $x=0$ and 0 everywhere else. The second impulsive function is $10^{-5}$ at $x=0$ and 0 everywhere else. In Fig. 2 we show the accessibility chart in the $(h, \alpha)$ plane. If both initial conditions lead to unstable chaotic behavior, we plot a black diamond. If both initial conditions lead to breathers that are periodic in $t$ as well as in $x$, we plot a blue dot. If both solutions are plane waves, we plot a yellow triangle. If the larger impulsive function leads to a breather and the smaller impulsive function leads to a plane wave, we plot an inverted red triangle. Finally, if both initial conditions lead to a cnoidal wave, we plot a green square. We see that there is a broad range of parameters from which the cnoidal waves are accessible from impulsive initial conditions. We also note that the parameter range over which stable cnoidal waves exist is significantly larger, but as $\alpha$ decreases, they no longer can be accessed from impulsive initial conditions.

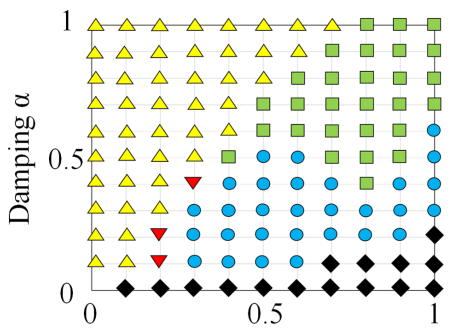

External pump $h$

Fig. 2. Accessibility chart in the $(h, \alpha)$ plane for a period 5.

In conclusion, we have demonstrated that the Lugiato-Lefever-equation for microresonators has stable cnoidal-wave solutions that are analogous to solitons and are accessible from impulsive, as well as cnoidal initial conditions. The spectrum of these solutions falls off exponentially with the mode number.

\section{References}

1. J. D. Jost, T. Herr, C. Lecaplain, V. Brasch, M. H. P. Pfeiffer, and T. J. Kippenberg, "Counting the cycles of light using a self-referenced optical microresonator," Optica 2, 706-711 (2015).

2. P. Del'Haye, A. Coillet, T. Fortier, K. Beha, D. C. Cole, K. Y. Yang, H. Lee, K. J. Vahala, S. B. Papp, S. A. Diddams, "Phase Coherent Link of an Atomic Clock to a Self-Referenced Microresonator Frequency Comb," ArXiv 1511,08103v1 (25 Nov. 2015)

3. K. J. Vahala, "Optical microcavities," Nature, 424, 839-846 (2004).

4. J. Roslund, R. M. Medeiros de Araujo, S. Jiang, C. Fabre and N. Treps, "Wavelength-multiplexed quantum networks with ultrafast frequency combs," Nat. Phot. 8, 109-112 (2014)

5. Y. K. Chembo and C. R. Menyuk, "Spatiotemporal Lugiato-Lefever formalism for Kerr-comb generation in whispering-gallery-mode resonators," Phys. Rev. A 87, 053852-1-5 (2013).

6. C. Godey, I. V. Balakireva, A. Coillet, and Y. K. Chembo, "Stability analysis of the spatiotemporal LugiatoLefever model for Kerr optical frequency combs in the anomalous and normal dispersion regimes," Phys. Rev. A 89, 063814-1-21 (2014).

7. P. F. Byrd and M. D. Friedman, Handbook of Elliptic Integrals for Engineers and Scientists (Springer-Verlag, 1971).

8. A. Matsko, A. Savchenkov, W. Liang, V. Ilchenko, D. Seidel, and L. Maleki, "Mode-locked Kerr frequency combs," Opt. Lett. 36, 2845-2847 (2011). 